\title{
Estudo bibliométrico da produção científica brasileira e internacional sobre gênero na educação médica
}

\section{Francisco José Passos Soares ${ }^{1}$, Debora C. Lopes do Nascimento' Gabriela Xavier Veiga ${ }^{1}$, Angélica Peixoto Teixeira ${ }^{1}$ e Ana Beatriz Machado Lessa ${ }^{1}$}

\author{
${ }^{1}$ Faculdade de Medicina Universidade Federal de Alagoas, Brasil \\ francisco passos01@hotmail.com; debora.nascimento@famed.ufal.br; \\ gabriela.veiga@famed.ufal.br; angelica.teixeira@famed.ufal.br; beatrizmlessa@gmail.com | \\ https://orcid.org/ 0000-0002-5872-2183; https://orcid.org/0000-0002-2735-4680; \\ https://orcid.org/0000-0002-1128-9044; https://orcid.org/0000-0003-3644-2348; \\ https://orcid.org/0000-0003-2161-5541
}

Resumo: Introdução: Para o enfrentamento aos estereótipos de gênero e princípios patriarcais, estruturais, presentes no currículo médico, as Diretrizes Curriculares Nacionais para os cursos de medicina orientam para a abordagem das necessidades de saúde, inclusive a pesquisa relacionada ao gênero. Objetivos: Analisar a evolução na produção científica relacionada a gênero, em três importantes periódicos de educação médica, dos Estados Unidos da América, da Europa e do Brasil, considerando a possiblidade de influência externa na pesquisa científica brasileira. Métodos: Pesquisa bibliográfica apoiada na teoria da neobibliometria, utilizando como indicadores bibliométricos: periódico; ano de publicação; temática; autoria; incidência das temáticas; metodologia. Resultados: semelhanças na abordagem temática entre os periódicos analisados, e atraso na pesquisa e baixo número de pesquisas nacionais originais, evidenciam a possibilidade de colonização da pesquisa e da educação médica brasileira; o protagonismo feminino na autoria é evidente e crescente; a pesquisa nacional deve, com originalidade, criar as condições para a transformação das práticas docentes, e de currículos, considerando a mais ampla visão sobre o papel da universidade, o trabalho médico, e as demandas sociais.

Palavras-chave: Saúde da Mulher; Medicina; Gênero e Saúde.

Bibliometric Study of Brazilian and International's Scientific Production on Gender in Medical Education

Abstract: Introduction: In the face of gender stereotypes and patriarchal, structural principles present in the medical curriculum, the National Curriculum Guidelines for medical courses guide the approach of health needs, including gender-related research. Goals: The evolution in gender-related scientific production were verified in three important medical education journals, from the United States of America, Europe, and Brazil, considering the possibility of external influence in Brazilian scientific research. Methods: Bibliographic research based on the theory of neobibliometry, using as bibliometric indicators: journal; year of publication; thematic; authorship; incidence of the themes; metodology. Results: similarities in the thematic approach between journals, and delay in research and a low number of original national studies, highlights the possibility of colonization of Brazilian medical research and education; the female protagonism in authorship is evident and growing; national research must, with originality, create the conditions for the transformation of teaching practices, and curricula, considering the broader view of the role of the university, medical work, and social demands.

Keywords: Women's Health; Medicine; Gender and Health.

\section{Introdução}

No estudo das variáveis relacionadas ao processo saúde-doença, sexo e gênero estão interligados, de modo que o espaço físico e os comportamentos sociais influem na fisiologia humana (McGregor et al., 2016). Inúmeros estudos sobre temas como farmacoterapia, doenças cardiovasculares, distúrbios do desenvolvimento e comunicação médico-paciente sugerem que sexo e gênero influenciam em estágios distintos de prevenção, diagnóstico, tratamento e prognóstico (Meulen et al., 2017). 
Isso explicita a amplitude de evidências científicas, relevantes, para a formação, a pesquisa e atuação de profissionais de saúde.

No currículo de Medicina, os assuntos relativos às mulheres são geralmente restritos aos aspectos biológicos, especificamente às funções reprodutivas e diferenças morfologicamente explícitas, tais como gravidez, contracepção, infertilidade, disfunções menstruais e câncer de mama; quanto aos homens, resumem-se às doenças do pênis e próstata, sendo essas as formas dominantes de diferenciação na assistência à saúde desses sujeitos (Lagro-Janssen, 2010).

Resultados de pesquisas conduzidas em homens, aplicadas no atendimento clínico de mulheres, contribuíram para disparidades de gênero na assistência à saúde (McGregor et al., 2016). Este fenômeno denominado andronormatividade, em que o homem serve como parâmetro, desconsidera o fato das diferenças fisiológicas das mulheres com efeitos nas respostas a doenças, como a síndrome coronariana aguda, e a medicamentos, podendo levar a tratamentos ineficazes ou a toxicidade a medicamentos (Lagro-Janssen, 2010). Embora o National Institutes of Health Revitalization Act, tenha exigido em 1993 a inclusão mandatória de mulheres e minorias étnicas nos ensaios clínicos, as diferenças nos resultados não são comumente reportadas de acordo com os grupos, bem como a amostra de pacientes pertencentes a essas populações, 20 anos após essa medida, persiste consistentemente pequena (Chen et al., 2014). Esse fato leva à insegurança nas informações contidas nas bulas, e ao fenômeno descrito como cegueira de gênero (LagroJanssen, 2010).

Os entraves relativos à inclusão de questões relacionadas ao gênero no ensino médico podem ser explicados pela teoria da colonização curricular definida como:

A constituição de um currículo é um processo social no qual convivem lado a lado fatores lógicos, epistemológicos, intelectuais, determinantes sociais menos "nobres" e menos "formais", tais como interesses, rituais, conflitos simbólicos e culturais, necessidades de legitimação e controle, propósitos de dominação dirigidos por fatores ligados à classe, à raça e ao gênero. Um currículo é constituído de conhecimentos considerados socialmente válidos. Há uma ação consciente de impedir também a validação de outros conhecimentos que se constituam como pertinentes, pois, estes são considerados com o estranhamento e o distanciamento para com o Outro, o colonizado. (Oliveira, 2018)

O Brasil tem se constituído culturalmente de forma colonizada com forte influência europeia e americana. No entanto, a presença africana e indígena, e mais recentemente de imigrantes de origem oriental, tem implicado em características próprias de saúde e adoecimento que precisam ser consideradas na reflexão sobre a formação médica e em saúde. A convivência com doenças persistentes e aquelas relacionadas ao estilo de vida moderno implica na necessidade de políticas orientadoras de formação para o trabalho em saúde distintas de países centrais, com ampla distribuição de renda.

Frente a essa realidade foi formulada a pergunta: quais as influências da evolução das produções externas, em educação médica, na pesquisa científica brasileira relacionada ao gênero?

Esse estudo teve como objetivo analisar a evolução na produção científica relacionada a gênero, em três importantes periódicos de educação médica, dos Estados Unidos da América, da Europa e do Brasil, considerando a possiblidade da influência externa na seleção temática da pesquisa científica brasileira.

\section{Metodologia}

Pesquisa bibliográfica, valendo-se da base de dados fornecida na área de pesquisa da página web específica dos periódicos analisados, no período de observação de 10 anos (2009-2019). 
Foi adotada como teoria norteadora a neobibliometria, a qual preocupa-se, além dos números, adotados como objetos de estudo, também com as teorias, métodos e amostras populacionais, caracterizando em profundidade os caminhos teóricos percorridos pelos pesquisadores, pela utilização de métodos complementares. (Silva et al., 2011)

Segundo Hayashi (2013), a bibliometria tem como uma de suas aplicações a "identificação de áreas de excelência, associações temáticas, interdisciplinaridade, redes de colaboração científica, temas emergentes e lacunas na produção do conhecimento científico".

Assume-se, nesta pesquisa, a parcialidade das informações, restringindo-se a três periódicos, considerando-se que a totalidade das publicações sobre gênero na educação médica é praticamente impossível de quantificar e qualificar em virtude da diversidade, do viés de cobertura, e da fragmentação e dificuldade de acesso às fontes desse conhecimento específico (Kobashi, 2004).

Os critérios para a seleção dos periódicos foram: importância científica para a formação médica; importância para a divulgação de pesquisas em cada região; vinculação a importantes associações responsáveis por agregar a comunidade regional de docentes e pesquisadores em educação médica. Foram selecionados os periódicos: Medical Teacher (MT), vinculado a An International Association for Medical Education ${ }^{1}$; Academic Medicine (AM), vinculado à Association of the American Medical Colleges²; Revista Brasileira de Educação Médica (RBEM), vinculada à Associação Brasileira de Educação Médica ${ }^{3}$.

Foram utilizados como indicadores bibliométricos: periódico (RBEM, MT, AM); temporalidade dos trabalhos (ano de publicação); temática (corpo biológico e corpo social); autoria (homem, mulher como autora principal, mulher como autora secundária); incidência das temáticas (técnicas pedagógicas, reflexão crítica); metodologia adotada (quantitativa, qualitativa, original ou revisão); aproximação temática entre os periódicos. $O$ cruzamento desses indicadores permitiu o aprofundamento das análises.

Foram considerados para análise os artigos científicos que incluiam descritores referentes a gênero nos títulos, resumos e palavras-chave.

As relações entre o ensino médico e gênero foram identificadas a partir de termos ou expressões constantes, em português e inglês: feminismo, fêmea, feminino, gênero, mulher, sexo, sexual, sexismo; feminism, female, feminine, gender, woman, women, sex, sexual, sexism.

Esses descritores foram pesquisados de maneira independente, por três pesquisadoras, nos resumos dos artigos dos três periódicos.

Foram excluídas da análise as seções "comentários" / "commentaries" ou "visão pessoal"/"personal view" mesmo que contivessem descritor relacionado a gênero. Além disso, foram excluídos os artigos que traziam esses descritores em análises exclusivas epidemiológicas nas quais não se exibia distinção dos resultados entre gêneros.

As categorias analíticas de gênero emergiram a partir das frequências em cada periódico servindo de substrato para a construção das tabelas, análise dos resultados e discussão. Foram observadas ainda as associações entre indicadores bibliométricos em cada periódico.

Para os artigos com autoria principal masculina e com autoria secundária feminina optouse por incluí-los em ambas as categorias. Os artigos com metodologia mista foram incluídos nas duas categorias, quantitativa e qualitativa. Em relação às temáticas escolheu-se aquela na qual o artigo encaixava-se mais, mesmo ele discursando sobre outro tema, secundariamente.

\footnotetext{
1 http://www.medicalteacher.org/MEDTEACH_wip/pages/home.htm

2 https://journals.Iww.com/academicmedicine/pages/default.aspx

$3 \mathrm{http}: / /$ www.scielo.br/scielo.php?script=sci_issues \&pid=0100-5502\&lng=pt\&nrm=iso
} 
A comparação dos resultados entre os periódicos restringiu-se às descrições de frequências simples ( $\mathrm{n}$ e \%), não sendo reconhecida a necessidade de aplicação de testes estatísticos comparativos.

Para organização dos dados e posterior análise, utilizou-se a planilha eletrônica Microsoft Excel, visando a apreciação de aspectos individuais bem como o cruzamento de dados que permitissem análises mais abrangentes.

A coleta de dados foi realizada em 2020. Sendo os dados coletados e analisados de domínio público, o estudo não foi submetido ao Comitê de Ética em Pesquisa com Seres Humanos. A gestão da instituição de vinculação dos pesquisadores foi informada sobre a natureza da pesquisa com o fim de obtenção de declaração de ciência e assentimento para com o desenvolvimento ético de todas as etapas.

\section{Resultados e Discussão}

Embora não seja possível partir de uma situação de completa objetividade (Rodney, 2016), visto que os conhecimentos sempre estão situados (Haraway, 2004), o currículo tem a capacidade de determinar o que é considerado "normal" (o homem, branco, atlético) ou periférico (Sharma, 2018). Tais concepções de validade e generalidade colocam de lado os contextos específicos históricos, políticos, sociais e culturais, inclusive no que tange a gênero na pesquisa científica na educação médica (Whitehead, 2015).

Nas narrativas presentes nas pesquisas médicas nota-se um padrão andronormativo, eurocêntrico, com supressão da localização epistêmica do autor, seu posicionamento nas estruturas de poder (Santiago, 2019).

A tabela 1 revela que há negligência do tópico gênero. Em relação ao total de publicações no período analisado, em todos os periódicos, apenas $4,76 \%$ dos artigos se encaixavam nos critérios de inclusão.

Tabela 1. Frequências de publicações científicas relacionadas a gênero, por ano, em relação ao número total de publicações nos períodos estudados, Maceió, 2021 (elaborada pelos autores).

\begin{tabular}{ccccccccccc}
\hline & \multicolumn{2}{c}{ Academic Medicine } & \multicolumn{3}{c}{ Medical Teacher } & \multicolumn{2}{c}{$\begin{array}{c}\text { Revista Brasileira } \\
\text { de Educação Médica }\end{array}$} \\
\hline Ano & $\begin{array}{c}\text { Publi- } \\
\text { cados }\end{array}$ & $\begin{array}{c}\text { Incluí- } \\
\text { dos }\end{array}$ & $\begin{array}{c}\text { Incluí- } \\
\text { dos } \\
\text { (total) }\end{array}$ & $\begin{array}{c}\text { Publi- } \\
\text { cados }\end{array}$ & $\begin{array}{c}\text { Incluí- } \\
\text { dos }\end{array}$ & $\begin{array}{c}\text { Incluí- } \\
\text { dos } \\
\text { (total) }\end{array}$ & $\begin{array}{c}\text { Publi- } \\
\text { cados }\end{array}$ & $\begin{array}{c}\text { Incluí- } \\
\text { dos }\end{array}$ & $\begin{array}{c}\text { Incluí- } \\
\text { dos } \\
\text { (total) }\end{array}$ \\
\hline 2009 & 247 & 14 & $5,67 \%$ & 231 & 9 & $3,90 \%$ & 85 & 1 & $1,18 \%$ \\
2010 & 286 & 13 & $4,55 \%$ & 206 & 7 & $3,40 \%$ & 73 & 0 & $0,0 \%$ \\
2011 & 232 & 20 & $8,62 \%$ & 226 & 12 & $5,31 \%$ & 73 & 0 & $0,00 \%$ \\
2012 & 243 & 10 & $4,12 \%$ & 240 & 15 & $6,25 \%$ & 124 & 1 & $0,81 \%$ \\
2013 & 285 & 6 & $2,11 \%$ & 246 & 18 & $7,32 \%$ & 70 & 3 & $4,29 \%$ \\
2014 & 280 & 7 & $2,50 \%$ & 148 & 8 & $5,41 \%$ & 67 & 1 & $1,49 \%$ \\
2015 & 264 & 12 & $4,55 \%$ & 160 & 5 & $3,13 \%$ & 76 & 1 & $1,32 \%$ \\
2016 & 257 & 19 & $7,39 \%$ & 173 & 2 & $1,16 \%$ & 93 & 1 & $1,08 \%$ \\
2017 & 276 & 12 & $4,35 \%$ & 193 & 2 & $1,04 \%$ & 70 & 1 & $1,43 \%$ \\
\hline
\end{tabular}




\begin{tabular}{cccccccccc}
\hline & \multicolumn{2}{c}{ Academic Medicine } & \multicolumn{3}{c}{ Medical Teacher } & \multicolumn{2}{c}{ Revista Brasileira } \\
de Educação Médica
\end{tabular}

Analisando individualmente os periódicos, a Academic Medicine apresentou o maior percentual de artigos relacionados a gênero (5,53\%), seguido por Medical Teacher $(4,46 \%)$, e RBEM $(3,12 \%)$. Observando-se a variação na frequência de publicações no período estudado, embora irregular para os três periódicos, a RBEM apresenta uma evolução importante, com publicação reduzida no período 2009 a 2017, e valores percentuais superiores aos demais periódicos em 2018. Academic Medicine mantém o padrão mais regular de publicação específica a gênero, enquanto Medical Teacher após publicar no triênio 2011-2012-2013 um número expressivo e crescente de artigos com temáticas referentes a gênero $(5,31 \%, 6,25 \%$ e $7,32 \%)$ apresentou redução progressiva e nenhuma publicação em 2019.

A tabela 2 explicita os principais conteúdos abordados nos periódicos descritos.

Tabela 2. Frequências de categorias temáticas relacionadas aos periódicos, Maceió, 2021 (elaborada pelos autores).

\begin{tabular}{|c|c|c|c|c|c|c|}
\hline \multirow{3}{*}{$\begin{array}{c}\begin{array}{c}\text { Periódicos e } \\
\text { categorias temáticas }\end{array} \\
\text { Práticas educativas e } \\
\text { sexualidade e gênero }\end{array}$} & \multicolumn{2}{|c|}{ Academic Medicine } & \multicolumn{2}{|c|}{ Medical Teacher } & \multicolumn{2}{|c|}{ RBEM } \\
\hline & $\mathbf{n}$ & $\%$ & $r$ & $\%$ & $\mathrm{n}$ & $\%$ \\
\hline & 33 & $22,6 \%$ & 10 & $11,76 \%$ & 8 & $26,62 \%$ \\
\hline $\begin{array}{l}\text { Discriminação e } \\
\text { violência de gênero }\end{array}$ & 25 & $17,12 \%$ & 2 & $2,35 \%$ & 5 & $18,51 \%$ \\
\hline Saúde da mulher & 1 & $0,68 \%$ & 1 & $1,17 \%$ & 3 & $11,11 \%$ \\
\hline $\begin{array}{l}\text { Qualidade de vida na } \\
\text { formação médica }\end{array}$ & 16 & $10,95 \%$ & 13 & $15,29 \%$ & 6 & $22,22 \%$ \\
\hline $\begin{array}{l}\text { Influência do gênero } \\
\text { sobre o cuidado }\end{array}$ & 12 & $8,21 \%$ & 15 & $17,64 \%$ & 3 & $\begin{array}{c}11,11 \\
\%\end{array}$ \\
\hline $\begin{array}{l}\text { Escolha de } \\
\text { especialidade médica }\end{array}$ & 9 & $6,16 \%$ & 14 & $16,47 \%$ & 1 & $3,7 \%$ \\
\hline $\begin{array}{l}\text { Técnicas pedagógicas } \\
\text { não relacionadas a } \\
\text { gênero }\end{array}$ & 21 & $14,38 \%$ & 18 & $21,17 \%$ & 0 & \\
\hline $\begin{array}{l}\text { Admissão na escola } \\
\text { médica }\end{array}$ & 5 & $3,42 \%$ & 7 & $8,23 \%$ & 0 & \\
\hline $\begin{array}{l}\text { Diversidade na escola } \\
\text { médica }\end{array}$ & 16 & $10,95 \%$ & 2 & $2,35 \%$ & 1 & $3,7 \%$ \\
\hline Outros & 8 & $5,47 \%$ & 3 & $3,52 \%$ & 0 & \\
\hline
\end{tabular}

Considerando as categorias de temas mais frequentes estudados observam-se distinções entre os periódicos. Pesquisadores americanos privilegiam estudos sobre: Práticas Educativas e Sexualidade e Gênero (19,7\%), a exemplo do artigo, Gender differences in academic productivity and leadership appointments of physicians throughout academic careers; Discriminação e Violência de Gênero (17,2\%), como Sexual Harassment in Medical Schools: The Challenge of Covert Retaliation as a Barrier to Reporting; e Técnicas Pedagógicas Não Relacionadas a Gênero (14,38\%), como Preventing and Managing Unprofessionalism in Medical School Faculties. 
Os pesquisadores europeus preocupam-se mais com Técnicas Pedagógicas Não Relacionadas à Gênero (21,17\%), a Influência do Gênero sobre o Cuidado (17,64\%), tal como "A gender-specific evaluation of a care-oriented curricular change in a Dutch medical school'; a Escolha da Especialidade Médica (16,47\%), a exemplo de "Medical career selection among newly graduated physicians in Madinah, KSA"; e Qualidade de Vida na Formação Médica (15,29\%), como "Exam anxiety in the undergraduate medical students of Taibah University".

Os pesquisadores brasileiros equilibram os interesses de pesquisa valorizando temas comuns aos americanos e europeus: Práticas Educativas e Sexualidade e Gênero $(29,62 \%)$ como "Jogos para capacitação de profissionais de saúde na atenção à violência de gênero", Qualidade de Vida na Formação Médica (22,22\%), exemplificado por "Prevalência de Burnout entre Médicos Residentes de um Hospital Universitário" e Discriminação e Violência de Gênero (18,51\%) "Assédio moral nas residências médica e não médica de um hospital de ensino". O que mais distingue a pesquisa nacional dos demais periódicos estudados é a ausência de estudos relacionados às técnicas pedagógicas, à admissão na escola, e escolha da especialidade médica, e o maior interesse sobre a saúde da mulher, temas provavelmente mais explorados em programas de pós-graduação em ensino na saúde, e publicados em formato de livro, em periódicos não analisados, ou ainda não publicados.

É fundamental que essas questões se façam presentes tanto na pesquisa quanto no ensino. Em 2002, a Organização Mundial de Saúde (OMS) assumiu o compromisso de promover igualdade e equidade de gênero na saúde, direcionando políticas específicas que visem a reparar as inequidades decorrentes dos papéis de gênero e de suas relações de poder desproporcionais. Esse objetivo deverá ser alcançado mediante análise sistemática, orientando os esforços a "planejamento, implementação, monitoramento e avaliação de políticas de saúde, programas, projetos e pesquisas", de modo que as questões de gênero passem a integrar o treinamento de profissionais de saúde, o que é essencial haja vista a posição privilegiada que esses indivíduos se encontrarão para impactar os problemas relacionados a gênero e sexualidade (World Health Organization, 2006).

De acordo com tais orientações, as Diretrizes Curriculares Nacionais do Curso de Graduação em Medicina (DCN), mencionam em seu artigo $5^{\circ}$ que:

$\mathrm{Na}$ Atenção à Saúde, o graduando será formado para considerar sempre as dimensões da diversidade biológica, subjetiva, étnico-racial, de gênero, orientação sexual, socioeconômica, política, ambiental, cultural e ética e demais aspectos que compõem o espectro da diversidade humana que singularizam cada pessoa ou cada grupo social [...] (Ministério da Educação, 2014).

No entanto, apesar de haver uma quantidade significativa de Projetos Pedagógicos Curriculares de cursos de Medicina que abordam questões de gênero, isso não se reflete na prática, já que se nota uma "visibilidade seletiva" aos aspectos patológicos e técnicos, em detrimento de uma abordagem que supere o exclusivamente biológico, realizando uma discussão interdisciplinar com as ciências humanas (Raimondi et al., 2020).

Como principal revista de educação médica brasileira, a RBEM, que publica artigos produzidos, em geral, por docentes de medicina, reflete a pequena representação desses temas e crítica gênero-sexual insuficiente na universidade, apesar da orientação específica nas DCN.

É fato, ainda, que as mulheres enfrentam diversas dificuldades em relação a seus colegas homens durante a atividade, recebendo menores salários e investimentos acadêmicos, além de menor celeridade na progressão de carreira, e da presença de obstáculos para publicação de suas pesquisas. Esta última representa um fator preponderante para a desigualdade de gênero nas posições de liderança no meio acadêmico (Sebo et al., 2020). É possível que com a ocupação crescente de mulheres nos cursos de medicina, e com as mudanças promovidas a partir das DCN, esse cenário venha a ser modificado. 
Há de se considerar, porém, que essa lacuna de autoria feminina tem sido reduzida ao longo do tempo. Foi observado que em seis revistas médicas de grande relevância a proporção de artigos com mulheres como autoras principais cresceu de $27 \%$ em 1994 para 37\% em 2014 (Filardo et al., 2016).

Os resultados dessa pesquisa demonstram o crescimento de publicações de autoria feminina em diversos temas, particularmente sobre gênero.

Nas publicações da Academic Medicine há maior envolvimento das mulheres $(75,3 \%)$ na pesquisa educacional relativa a gênero, com especificidades, predominando as preocupações com a temática social, e a reflexão crítica, embora o uso das metodologias quantitativas supere as metodologias qualitativas.

No periódico europeu, Medical Teacher, observa-se equilíbrio entre homens e mulheres $(50,5 \times 49,4 \%)$ como autores principais, com temáticas relacionadas ao corpo social, porém com predominância de investigação de métodos pedagógicos e uso de metodologias quantitativas. As pesquisas orientadas pela reflexão crítica são realizadas em maioria por homens.

A RBEM, o periódico com menor volume de publicações sobre gênero, demonstra um padrão de associações similar ao do periódico Academic Medicine, com preocupações sobre o corpo social, reflexões críticas, e uso de metodologias qualitativas, e autoria predominante feminina $(58,6 \%)$.

Fica claro que o interesse feminino pelas questões de gênero supera o masculino, considerando que sempre coube às mulheres debater e questionar as estruturas patriarcalistas que as oprimem, desde o início do movimento feminista no século XIX, com as bandeiras de direito a voto, estudo, propriedade, trabalho e herança (Silva \& Silva Junior, 2014).

Em relação ao contexto específico da publicação científica, as mulheres são mais propensas a apoiar a implementação de políticas pelas revistas no sentido de evitar a negligência das diferenças de sexo e gênero no desenho das pesquisas e na comunicação científica de forma geral, introduzindo essas considerações como instrução para os autores (Heidari et al., 2017). Além de, conforme descrito, as mulheres constituírem maioria nas publicações sobre esta temática.

Quanto às temáticas dos artigos incluídos, 95,73\% desses categorizam-se como corpo social, sendo 95,89\% da Academic Medicine, 96,47\% da Medical Teacher e 92,59\% da RBEM. Isso se deu, principalmente, em função do critério de exclusão adotado nessa pesquisa, que não incluía artigos com análises exclusivas epidemiológicas nas quais não se exibia distinção dos resultados entre gêneros. Além disso, a temática sexo/gênero é, primariamente, de cunho social.

Como revistas de educação médica, uma boa proporção dos artigos incluídos versava sobre técnicas pedagógicas (32,55\%) no total, sendo 19,86\% na Academic Medicine, $57,64 \%$ na Medical Teacher e $22,22 \%$ na RBEM. E, para além do cunho puramente pedagógico, as reflexões críticas prevaleceram no total $(67,45 \%)$, com $80,14 \%$ na Academic Medicine, 42,36\% na Medical Teacher e 77,78\% na RBEM, observando a valorização de tais reflexões sobre essa temática.

As reflexões levantadas pela RBEM assemelham-se muito com os periódicos internacionais, a exemplo de discussões sobre empatia (Empathy in Korean medical students: Findings from a nationwide survey, Medical Teacher, 2015; Measurement of Empathy Among Japanese Medical Students: Psychometrics and Score Differences by Gender and Level of Medical Education, Academic Medicine, 2009; Análise dos Níveis de Empatia de Estudantes de Medicina, RBEM, 2018) e depressão e ansiedade (Social anxiety in medical students: Implications for communication skills teaching, Medical Teacher, 2009; Depressive Symptoms in Medical Students and Residents: A Multischool Study, Academic Medicine, 2009; Fatores Associados à Depressão e Ansiedade entre Estudantes Universitários da Área da Saúde de um Grande Centro Urbano do Nordeste do Brasil, RBEM, 2019). 
Apenas dois artigos na RBEM foram considerados abordar problemas intrinsecamente nacionais: "Influência de Políticas de Ação Afirmativa no Perfil Sociodemográfico de Estudantes de Medicina de Universidade Brasileira" e "Incorporando a Competência Cultural para Atenção à Saúde Materna em População Quilombola na Educação das Profissões da Saúde", ambos de 2018.

As semelhanças entre a RBEM e demais periódicos analisados, o atraso na pesquisa sobre gênero e o baixo número de pesquisas originais evidenciam a possibilidade de colonização da educação médica brasileira. Apesar disso, esses temas têm grande relevância em qualquer contexto e, independentemente da influência externa, são de interesse para evidenciar as características gerais e diferenças locais.

Quanto à metodologia empregada pelos artigos selecionados, na Academic Medicine e na Medical Teacher, foi observada maioria de artigos quantitativos, com $55,48 \%$ e $80,23 \%$, respectivamente. No entanto, na RBEM, $11,11 \%$ dos trabalhos pertenciam a esta categoria, predominando a qualitativa, com $77,77 \%$. Os artigos com metodologias originais e de revisão foram pouco representativos em todas as revistas.

Como limitações do método bibliométrico, a pesquisa sobre gênero, no Brasil, não se esgota com a publicação em periódicos sobre educação médica, sendo necessário ampliar a pesquisa com a verificação da produção científica em outros meios, como os repositórios de teses e dissertações, e livros.

\section{Conclusões}

O interesse pela temática relacionada a gênero entre educadores médicos no Brasil é tardio, iniciando somente em 2013 e alcançando maior impulso a partir de 2018. Embora os pesquisadores brasileiros demonstrem equilíbrio nas escolhas temáticas gerais, similares aos dos pares americanos e europeus, o padrão de predominante autoria feminina, abordagem principal de temas relacionados ao campo social, e uso principal de reflexões críticas e metodologias qualitativas de exploração, assemelha-se ao americano.

A distinção da pesquisa nacional sobre gênero é observada na menor produção científica, e ausência de estudos em determinadas categorias temáticas. Para a distinção positiva deve-se considerar a presença de temas e métodos pedagógicos que impulsionem a reflexão sobre a compreensão histórica, contextualizada, relacionada à formação étnica $e$ cultural, própria à diversidade brasileira, com repercussão nas formas de adoecimento, e de enfrentamento às contradições no mundo do trabalho, e na sociedade. A pesquisa nacional deve criar as condições para a transformação das práticas docentes, e de currículos, considerando a mais ampla visão sobre o papel da universidade, o trabalho médico, e as demandas sociais.

\section{Referências}

Chen Jr, M. S., Lara, P.N.,Dang, J.H.T., Paterniti, D.A. \& Kelly, K. (2014). Twenty years post-NIH Revitalization Act: Enhancing minority participation in clinical trials (EMPaCT). Cancer, (3), 1091-1096. http://dx.doi.org/10.1002/cncr.28575

Colling, A. M. (2011). As primeiras médicas brasileiras: Mulheres à frente de seu tempo. Fronteiras, 13(24), 169-183.

Filardo, G., Da Graça, B., Sass, D. M., Pollock, B. D., Smith, E. B. \& Martinez, M. A. (2016). Trends and comparison of female first authorship in high impact medical journals: Observational study (1994-2014). Bmj. http://dx.doi.org/10.1136/bmj.i847

Haraway, D. (2004). Gênero para um dicionário marxista: a política sexual de uma palavra. Cadernos Pagu, 22, 201-246.

Hayashi, C. R. M. (2013). Apontamentos sobre a coleta de dados em estudos bibliométricos e cientométricos. Filosofia e Educação, 5(2), 89-102. 
Heidari, S., Babor, T. F., De Castro, P., Tort, S. \& Curno, M. (2017). Equidade de sexo e gênero na pesquisa: Fundamentação das diretrizes sager e uso recomendado. Epidemiologia e Serviços de Saúde, 26(3), 665-676. http://dx.doi.org/10.5123/s1679-49742017000300025

Kobashi, N. Y. (2006). Institucionalização da pesquisa científica no Brasil: Cartografia temática e de redes sociais por meio de técnicas bibliométricas. TransInformação, 18(1), 27-36.

Lagro-Janssen, T. (2010). Gender and sex: issues in medical education. Undergraduate Medical Education, 29(1), 48-53.

McGregor, A. J., Núñez, A., Barron, R., Casanova, R. \& Chin, E.L. (2016). Workshop summaries from the 2015 Sex and Gender Medical Education Summit: utilization of sex and gender based medical education resources and creating student competencies. Biology of Sex Differences, 7(1), 11-17. http://dx.doi.org/10.1186/s13293-016-0092-8.

van der Meulen, F. V., Fluit, C.,Albers, M., Laan, R. \& Lagro-Janssen, A. (2017). Successfully sustaining sex and gender issues in undergraduate medical education: a case study. Advances In Health Sciences Education, 22(5), 1057-1070. http://dx.doi.org/10.1007/s10459$\underline{016-9742-1}$

Mohanty C. (2003). Feminism without borders decolonizing theory, practicing solidarity. Duke University Press.

Oliveira, R. P. (2018). Paulo Freire e ação cultural de descolonizar o currículo da educação do campo. Revista Panorâmica On-line, 24, 308-317.

Perez, O. \& Ricoldi, A. (2019). A quarta onda feminista: Interseccional, digital e coletiva. Anais do Quinto Congresso Latino-americano de Ciência Política (ALACIP). Associação Mexicana de Ciência Política e o Tecnológico de Monterrey.

Raimondi, G. A., de Abreu, Y.R., Borges, I.M., da Silva, G.B.M., Hattori, W.T. \& Paulino, D.B. (2020). Gênero e Sexualidade nas Escolas Médicas Federais do Brasil: uma Análise de Projetos Pedagógicos Curriculares. Revista Brasileira de Educação Médica, 44(2), e046. http://dx.doi.org/10.1590/1981-5271v44.2-20190050.ing

Resolução nº 3, de 20 de junho de 2014. Institui Diretrizes Curriculares Nacionais do Curso de Graduação em Medicina e dá outras providências. Ministério da Educação.

Rodney, R. (2016). Decolonization in health professions education: Reflections on teaching through a transgressive pedagogy. Canadian Medical Education Journal, 7(3), 10-18. http://dx.doi.org/10.36834/cmej.36840

Santiago, A. R.,de Carvalho, J.C., Barros, R.C.S. \& da Silva, R.S. (2019). Descolonização do conhecimento no contexto afro-brasileiro. Editora UFRB.

Scheffer, M. C. \& Cassenote, A. J. (2013). A feminização da medicina no Brasil. Revista de Bioética, 2(21), 268-277. http://www.scielo.br/pdf/bioet/v21n2/a10v21n2.pdf

Sebo, P., Maisonneuve, H. \& Fournier, J. P. (2020). Gender gap in research: a bibliometric study of published articles in primary health care and general internal medicine. Family Practice, 37(3), 325-331. http://dx.doi.org/10.1093/fampra/cmz091

Sharma, M. (2018). 'Can the patient speak?': Postcolonialism and patient involvement in undergraduate and postgraduate medical education. Medical Education, 52(5), 471-479. http://dx.doi.org/10.1111/medu.13501.

Silva, C. \& Silva Junior, C. (2014). Elas em foco: A produção sobre a mulher e questões de gênero na Universidade Federal de Alagoas. Anais do Oitavo Encontro da Rede Feminista Norte e Nordeste de Estudos e Pesquisas sobre Mulher e Relações de Gênero. Universidade Federal Rural de Pernambuco.

Silva, M. R., Hayashi, C. R. M. \& Hayashi, M. C. P. I. (2011). Análise bibliométrica e cientométrica: desafios para especialistas que atuam no campo. Revista de Ciência da Informação e Documentação, 2(1), 110-129.

Whitehead, C. R. (2015). On gunboats and grand pianos: Medical education exports and the long shadow of colonialism. Advances In Health Sciences Education, 21(1), 1-4. http://dx.doi.org/10.1007/s10459-015-9660-7

World Health Organization (2006). Integrating gender into the curricula for health professionals: meeting report. 